\title{
Good's syndrome twelve years after thymectomy due to thymoma. A case study
}

\author{
MARTA ADAMCZYK-KORBEL $L^{l}$, ROBERT KIESZKO ${ }^{l}$, PAWEŁ KRAWCZYK ${ }^{1}$, IWONA HOMA \\ RODRYG RAMLAU ${ }^{2,3}$, JANUSZ MILANOWSKI ${ }^{1,4}$
}

${ }^{1}$ Pneumonology, Oncology and Allergology Department Medical University of Lublin, Poland

${ }^{2}$ Department of Cardio-Thoracicsurgery, the University of Medical Sciences, Poznan, Poland

${ }^{3}$ Wielkopolska Center of Pulmonology and Thoracosurgery of Eugenia and Janusz Zeyland, Poznan, Poland

${ }^{4}$ Institute of Agricultural Medicine in Lublin, Poland

\begin{abstract}
The case of a 63-year-old man suffering from the immunodeficiency syndrome developed 12 years after thymectomy due to thymoma is reported. In our patients, the most frequently manifested symptoms resulted from recurrent infections of the upper and lower respiratory tract. In chest X-ray and computed tomography examination, bilateral ground-glass opacities, diffuse areas of infiltrates and bronchiectases are observed. The immunological analysis revealed an almost undetectable level of immunoglobulins in blood plasma and a very low percentage and number of peripheral blood $B$ lymphocytes. The inversion of the CD4/CD8 ratio and the presence of a large population of the CD4 and CD8 double-positive lymphocytes are also observed in peripheral blood. Based on clinical symptoms, Good's syndrome was diagnosed. This syndrome was observed very rarely in patients after thymectomy and occurred in the course of thymoma.
\end{abstract}

Key words: Good's syndrome, thymectomy, immunodeficiency, T-lymphocytes, B-lymphocytes.

(Centr Eur J Immunol 2013; 38(4): 505-510)

\section{Introduction}

Good's syndrome (GS) was described for the first time in 1954 [1]. Good's syndrome is a rare affection/condition occurring in adult people (to date fewer than 100 cases have reported in the literature). This is a rare case of thymoma, accompanied by the state of immunodeficiency, which is usually characterized by hypogammaglobulinemia and a low number of peripheral B-cells. CD4+ T-lymphopenia and the inverted CD4/CD8 T-cell ratio may also be present [2]. A representative patient affected by GS is 50 to 70 years old and develops recurrent bronchitis, sinusitis, or pneumonia, which may be complicated by bronchiectasis. Systemic symptoms such as weight loss, fatigue, chronic diarrhoea and dermatitis are common. In the rare cases, thymectomy does not improve the patient's condition in dealing with hypogammaglobulinaemia and other symptoms resulting from the immunological disturbances $[3,4]$.

It has not been explained yet what the cause of the immunological deficiency in the course of GS is. The case reported about immunological disturbances which had appeared after thymectomy. The results of these studies may be helpful in elucidating some aspects of the pathogenesis of GS.

\section{Case report}

In August 2001, a 63-year-old man with a three-year history of recurrent lung infections was admitted to the Department of Pneumonology, Oncology and Allergology, Medical University of Lublin, with the symptoms manifested as haemoptysis, fever, productive cough and progressive dyspnoea. The patient also reported a weight loss and fatigue for 4 weeks prior to the admission. In 1991, he had undergone thymectomy due to mixed epithelial and lymphocytic thymoma. The chest X-ray showed infiltrates in the superior and middle fields of the left lung and diffuse pleural adhesions. These lesions correlated with suppression of the vesicular murmur above the left lung and the bilateral basal inspiratory crackles, which was noticeable in the chest examination. Bilateral ground-glass opacities and diffuse areas of condensation as well as infiltrates in the left lung with the mediastinal displacement dominated on the computed tomography scan (Fig. 1A). The bronchoscopy and histological analysis of the material from endobronchial biopsy demonstrated a chronic bronchitis image with mild epithelial dysplasia. Pulmonary function tests revealed a decrease in respiratory reserve of a mixed character, i.e. forced vital capacity (FVC) $-2.94 \mathrm{~L}$ ( $76 \%$ of predicted normal values), forced expiratory volume in one

Correspondence: Iwona Homa, MSc, Pneumonology, Oncology and Allergology Department, Medical University of Lublin, Jaczewskiego 8, 20-950 Lublin, Poland, tel. +48 8172442 93, fax +48 8172448 23, e-mail: iwona.homa@wp.pl 

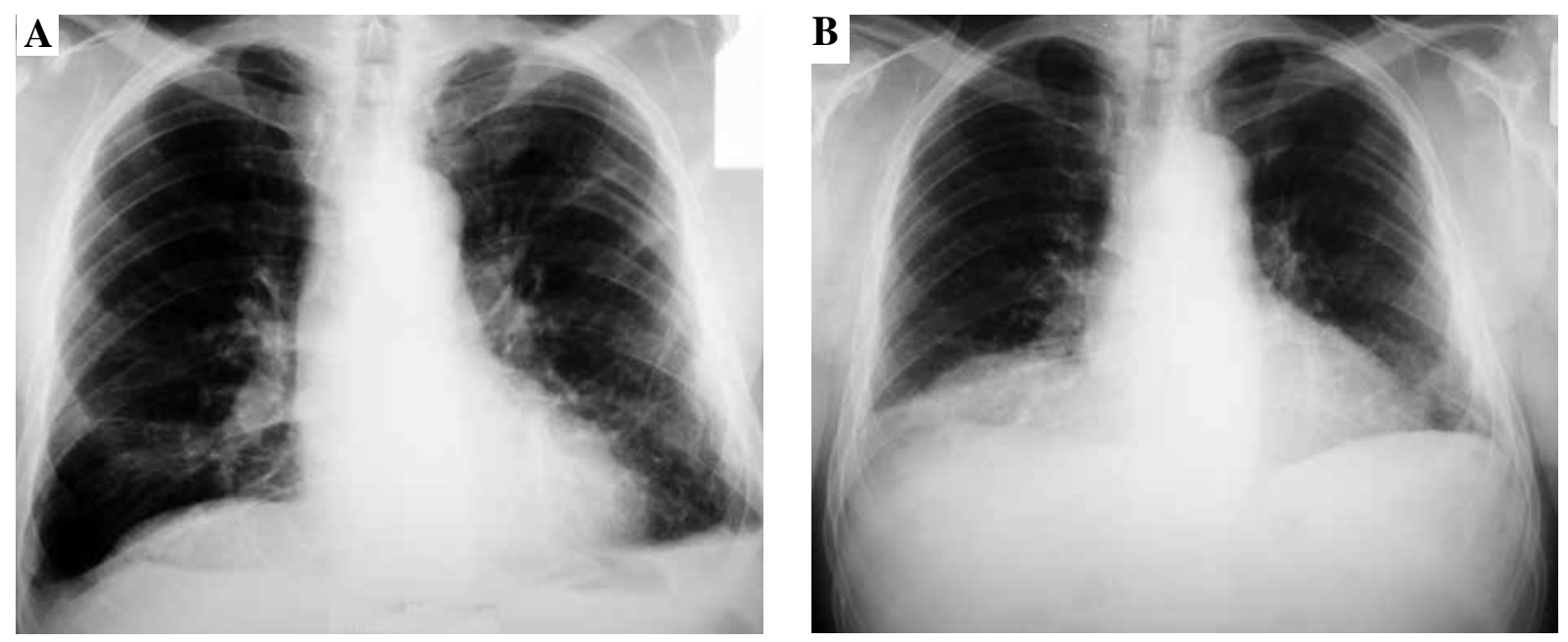

Fig. 1. A) Chest X-ray performed in March 2001 showing inflammatory changes in the lungs, B) Chest X-ray performed in February 2011 showing inflammatory changes in the lungs

second $\left(\mathrm{FEV}_{1}\right)-2.00 \mathrm{~L}(68 \%$ of $\mathrm{PV})$, and $\mathrm{FEV}_{1} / \mathrm{FVC}-$ $89 \%$ of the predictive normal value. The respiratory failure was not observed.

The microbiological analysis of the bronchoaspirate revealed the presence of Citrobacter braakii and Candida sp. The cultures of sputum yielded Streptococcus $\alpha$ and Candida sp. The results of other laboratory tests demonstrated a low level of total serum protein $(4.9 \mathrm{~g} / \mathrm{dl})$ due to an extreme reduction in the percentage and the level of $\beta$ - and $\gamma$-globulins (2.9\% - reference ranges: $8-17 \%$ and

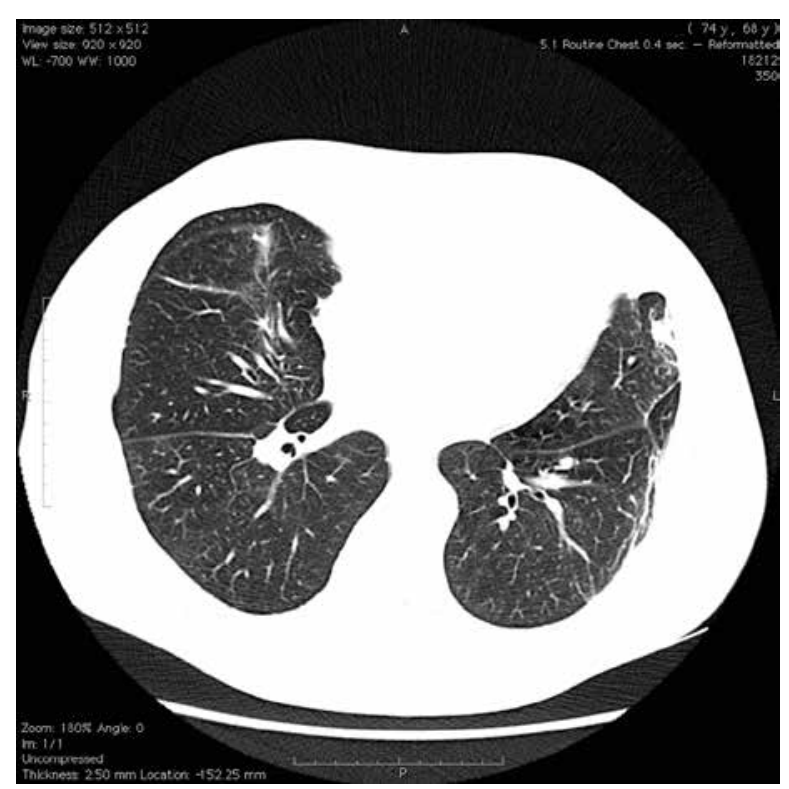

Fig. 2. CT scan showing bronchiectases, pulmonary infiltrations, ground-glass opacities and pleural deformations in Good's syndrome patient
$3.3 \%$ - reference ranges: $9-22 \%$, respectively). On the day of admission, the examination of the serum $\gamma$-globulin level was performed and an extremely low level of IgG (9 mg/dl reference ranges: $650-1600 \mathrm{mg} / \mathrm{dl}$ ) was found.

On the basis of the above examinations and laboratory tests, Good's syndrome (GS) was diagnosed. Due to the applied antibiotic therapy, a partial response with an improvement in respiratory symptoms and with a reduction in the lung infiltrations was observed on the chest radiography. Every four weeks, the patient has received an intravenous immunoglobulin (IVIG) infusion and each time it was accompanied by serum Ig level measurement. In the follow-up examinations, he maintained the satisfying IgG level, but IgA and IgM levels were very low. The symptoms have disappeared partially. Therefore, during the course of the disease in our patient we observed recurrent pneumonia (Fig. 1B) and bronchiectasis (Fig. 2). However, 22 years after thymectomy the patient remained in good condition.

After the absolute leucocytosis normalisation in the course of antibiotic treatment, the cytometric analysis of the peripheral blood lymphocyte subtypes was performed and repeated twice within 14 months from the disease diagnosis (Table 1, Fig. 3A-G). The results were compared with those obtained for a group of seven age-related healthy individuals. For the GS patient, the obtained results were characterised by the almost total absence of B-cells and a low percentage and number of helper (CD4+) T-lymphocytes (Th-lymphocytes) as well as a high percentage and number of cytotoxic/suppressor (CD8+) T-cells. The presence of the CD4 and CD8 double-positive lymphocytes and the small percentage of CD8 and CD28 double-positive cells was observed. Large CD4+ CD8+ CD3+ lymphocyte population has been on the constant level regardless of applied treatment and disease duration. Almost 
Table 1. Number of peripheral blood leucocytes and flow cytometry analysis of lymphocytes' subpopulation in healthy donors and Good's syndrome (GS) patient

\begin{tabular}{|c|c|c|c|c|}
\hline & $\begin{array}{c}\text { Healthy donors } \\
\text { (mean value } \pm \text { SD) }\end{array}$ & $\begin{array}{c}\text { GS patient before } \\
\text { immunological } \\
\text { treatment }\end{array}$ & $\begin{array}{c}\text { GS patient during } \\
\text { immunological } \\
\text { treatment }\end{array}$ & $\begin{array}{c}\text { GS patient in } \\
\text { September } 2002\end{array}$ \\
\hline Leucocytes (cells/ $\mu \mathrm{l})$ & $6340 \pm 1716 \%$ & 5900 & 9300 & 7900 \\
\hline Lymphocytes $(\% ;$ cells $/ \mu \mathrm{l})$ & $25.70 \pm 2.54 \%$ & $25.30 ; 1493$ & $25.80 ; 2399$ & $23.20 ; 1833$ \\
\hline CD19+ cells $(\% ;$ cells $/ \mu \mathrm{l})$ & $10.51 \pm 1.41 \%$ & $0.64 ; 10$ & $0.37 ; 9$ & $0.79 ; 1448$ \\
\hline CD3+ cells $(\% ;$ cells $/ \mu \mathrm{l})$ & $65.20 \pm 12.11 \%$ & $84.26 ; 1258$ & $85.39 ; 2062$ & $81.11 ; 1486$ \\
\hline CD4+ cells $(\% ;$ cells $/ \mu \mathrm{l})$ & $40.19 \pm 7.19 \%$ & $26.56 ; 396$ & $20.81 ; 499$ & $24.42 ; 447$ \\
\hline CD8+ cells $(\% ;$ cells $/ \mu \mathrm{l})$ & $35.3 \pm 8.76 \%$ & $44.42 ; 663$ & $44.79 ; 1075$ & $43.49 ; 797$ \\
\hline CD4+ CD8+ CD3+ cells $(\% ;$ cells $/ \mu \mathrm{l})$ & $1.29 \pm 0.53 \%$ & $16.44 ; 245$ & $22.29 ; 535$ & $18.06 ; 331$ \\
\hline CD4/CD8 ratio & $1.24 \pm 0.49 \%$ & 0.60 & 0.47 & 0.56 \\
\hline NK cells $(\% ;$ cells $/ \mu \mathrm{l})$ & $22.7 \pm 12.52 \%$ & $10.24 ; 9$ & $12.23 ; 293$ & $9.06 ; 166$ \\
\hline CD45RO+ of CD4+ cells ( $\%$; cells $/ \mu \mathrm{l})$ & $66.74 \pm 10.80 \%$ & $84.51 ; 1261$ & $96.66 ; 2319$ & $92.71 ; 1691$ \\
\hline CD45RO+ of CD8+ cells $(\%$; cells $/ \mu \mathrm{l})$ & $36.88 \pm 12.80 \%$ & $47.75 ; 713$ & $54.41 ; 1305$ & $49.96 ; 911$ \\
\hline CD45RA+ of CD8+ cells $(\% ;$ cells $/ \mu \mathrm{l})$ & $73.94 \pm 9.08 \%$ & $65.02 ; 970$ & $62.52 ; 1500$ & $64.45 ; 1181$ \\
\hline CD28+ of CD8+ cells $(\% ;$ cells $/ \mu \mathrm{l})$ & $43.8 \pm 11.05 \%$ & $31.61 ; 472$ & $14.91 ; 358$ & $22.64 ; 415$ \\
\hline
\end{tabular}

all helper T-lymphocytes and half of CD8+ T-cells were the memory cells with CD45RO antigen on the surface. The remaining percentage of $\mathrm{CD} 8+\mathrm{T}$-cells expressed CD45 RA antigen (Table 1).

\section{Discussion}

The thymus remains one of the least understood organs in the human body. It plays an important role in the T-cell maturation and in the elimination of lymphocyte autoreactive clones. After interaction with antigen presenting cells (e.g. dendritic cells) and epithelial cells, thymocytes undergo differentiation into T-lymphocytes. Immature thymocytes rearrange genes for $\alpha$ and $\beta$ or $\delta$ and $\gamma$ chains of T-cell receptor (TCR); first they become CD4 and CD8 double-negative early thymocytes, and then CD4 and CD8 double-positive intermediary thymocytes. Mature thymocytes differentiate into CD4 or CD8 single-positive T-lymphocytes. Peripheral memory T-cells express antigens (e.g. CD28) indispensable in the correct humoral and cellular immunological response. The thymus' involution starts in adolescence, but it is possible that T-cells mature in the thymus of adults. In adults, the endocrinological function of thymus may also be important $[5,6]$.

Pathologically, thymoma, the most common primary tumour of the anterior mediastinum is an epithelial neoplasm characterised by an admixture of epithelial cells and mature lymphocytes $[7,8]$. Immunodeficiency in an adult-onset thymoma is characterised by low numbers of B-cells or even their absence in the peripheral blood, hypogammaglobulinaemia, and, in some cases, by the defects in cell-mediated immunity with CD4+ T-lymphopenia as well as by the inverted CD4/CD8 T-cell ratio [2]. Its pathogenesis is still not entirely understood. Due to the presence of B- and Th-cell lymphopenia and the frequent coexistence of a low number of peripheral blood eosinophiles, a bone marrow defect is suggested [3].

This hypothesis might be explained by the interdependence between thymoma and autoimmune diseases. The studies confirmed that autoantigenic epitopes were expressed on neoplastic epithelial cells. This results in a positive selection of the helper T-cells maturing in tumours or sensitisation of the developing thymocytes or recirculating T lymphocytes. This process is observed, for instance, in myasthenia gravis with thymoma, where T-cell clones against muscle acetylcholine receptor express CD4 and CD $8 \alpha$ antigens and have a Th0 cytokine profile, producing interleukin 4 (IL-4) and interferon $\gamma($ IFN- $\gamma)$ [9]. It is possible that a subset of Th-cells in thymoma may also suppress the development of erythroid precursors in the pure red cell aplasia (PRCA) and the maturation of B-lymphocytes in the immunodeficiency syndromes [1012]. The failure to produce B-lymphocytes occurs on the stem cell level and pre-B-cells could not be found in the bone marrow. Waldmann et al. showed the presence of suppressor T-cells in the blood of patients with immuno- 
A

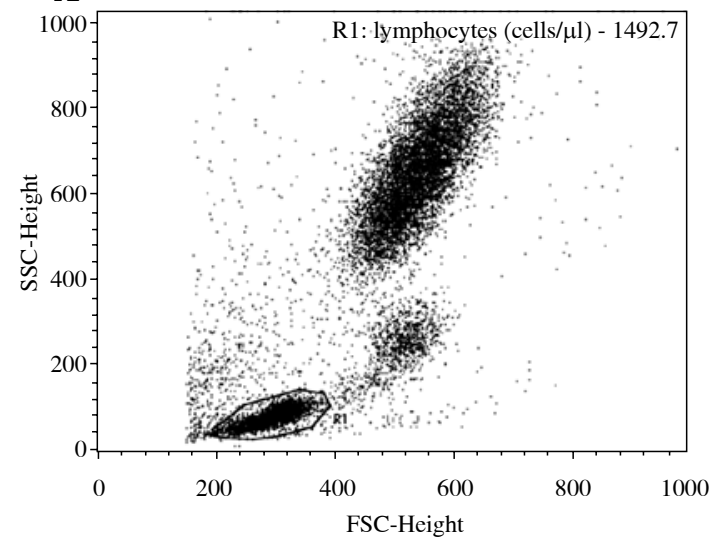

C

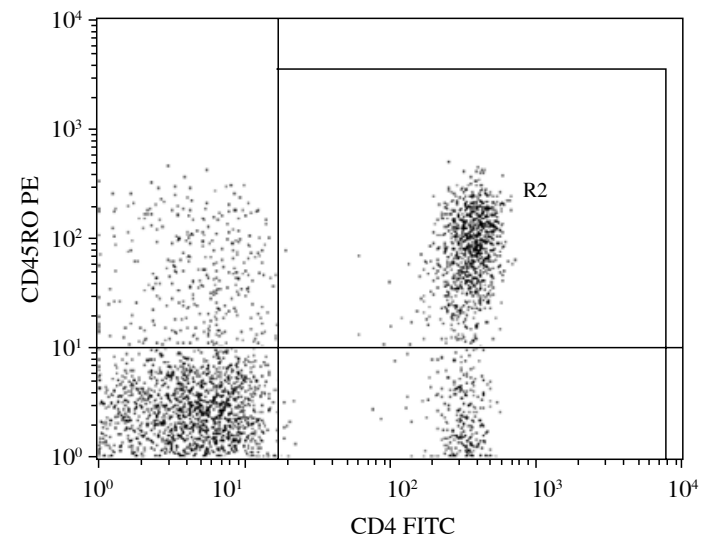

$\mathbf{E}$

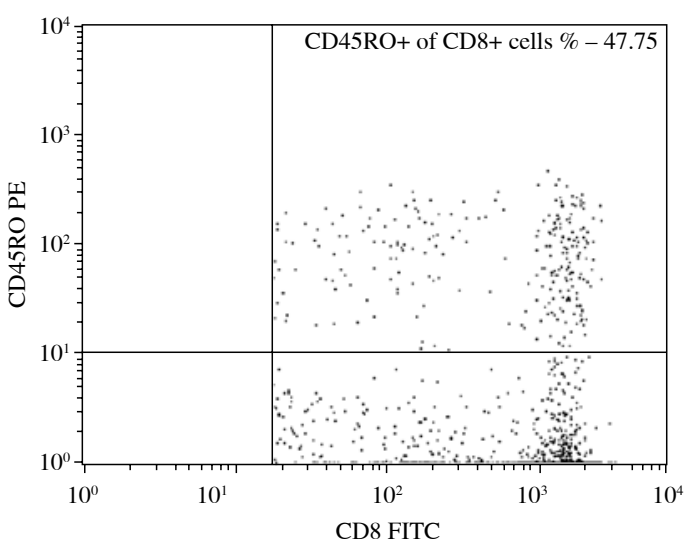

G

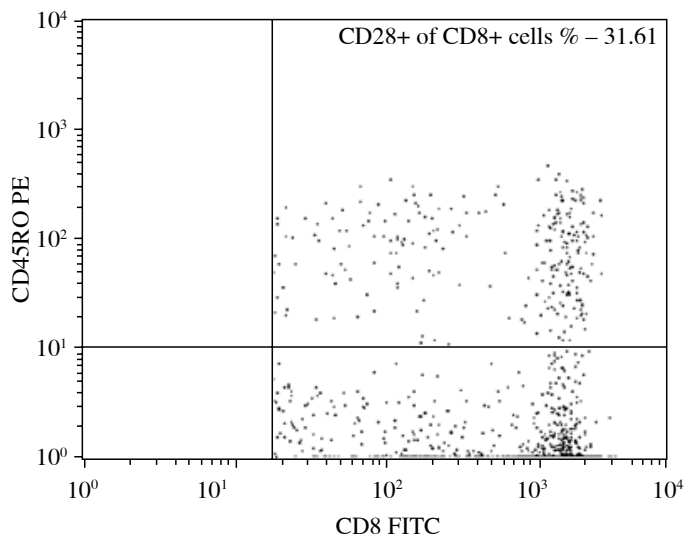

B

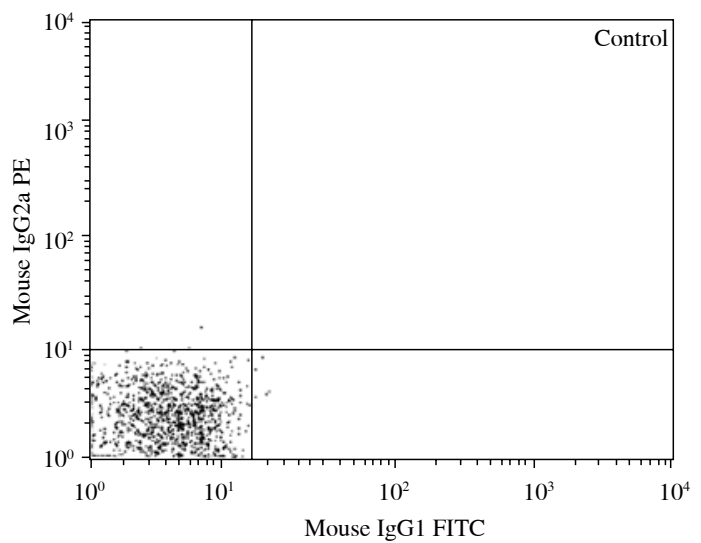

D

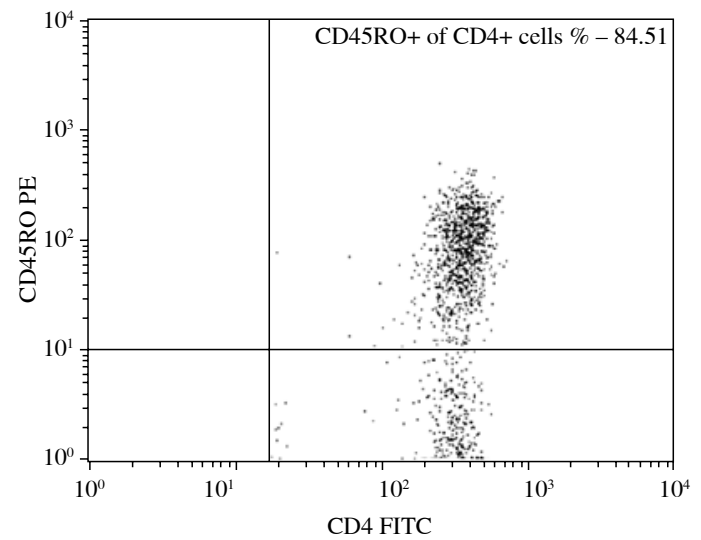

F

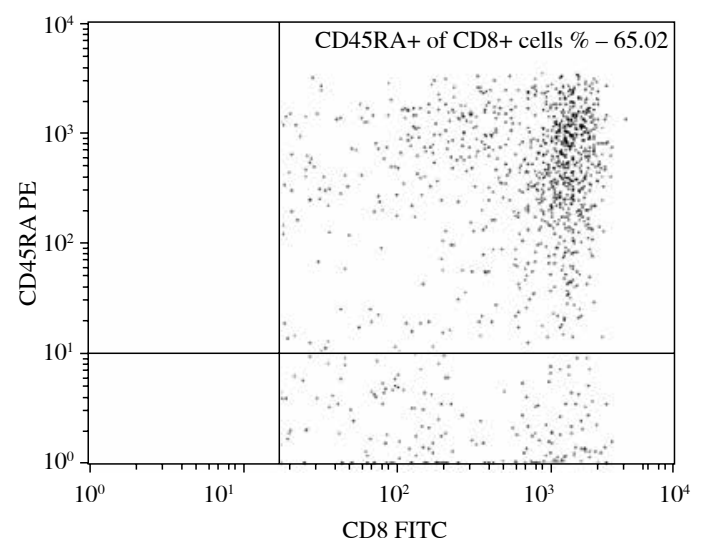

Fig. 3A-D. Representative three-colour flow cytometry analysis of lymphocytes subpopulations in Good's syndrome patient: A - Dot plot based on FSC and SSC parameters - lymphocytes are gated in R1; B - Control dot plot (immunostaining with monoclonal mouse antibody against IgG1 and IgG2a); C - Dot plot for the CD4-positive cells selection which are gated in R2 and further analysed in next dot plots (D-G); D - Dot plot for the percentage of double-positive CD4 and CD45RO lymphocytes; E Dot plot for the percentage of double-positive CD8 and CD45RO lymphocytes; $\mathrm{F}$ - Dot plot for the percentage of double-positive CD8 and CD45RA lymphocytes; G - Dot plot for the percentage of double-positive CD8 and CD28 lymphocytes 
deficiency associated with thymoma. Co-culture of normal B-lymphocytes and T-cells of hypogammaglobulinemic patients resulted in the decrease in immunoglobulin synthesis [13-15]. The relationship between thymoma, T-cell dysfunction and abnormalities of haematopoietic stem cells remains conjectural, especially since thymectomy has little, if any, effect on the immunological disturbances development and the coexisting condition in the patient with the acquired immunodeficiency [3]. Moreover, in some cases, the symptoms manifested themselves after the removal of the thymoma [12].

Hypogammaglobulinaemia and B-lymphopenia develop in 3-6\% of patients with thymoma [3] and this association is commonly referred to as GS. Even though a formal diagnostic definition of GS has not been developed, it is classified as a diagnostic entity ("immunodeficiency with thymoma") separate from common variable immunodeficiency (CVID) [16].

In the case of our patient, the symptoms of immunodeficiency appeared as late as 10 years after thymectomy due to thymoma. Moreover, at the moment of the thymoma diagnosis, the patient manifested no immunodeficiency except for the symptoms resulting from the compression of adjacent structures. The diagnosis of recurrent infections revealed that they might have been caused by a considerable decrease in the B-cell number and by an undetectable level of immunoglobulins. The presence of a large population of CD4 and CD8 double-positive cells in the peripheral blood and other changes in T-lymphocyte subpopulations suggested the cause of immunodeficiency in our patient. Because of the abnormal maturation and differentiation of T-cells in thymoma and after thymectomy, the question arises whether the hypothesis concerning the development of the autoreactive clones of T-lymphocytes in thymoma is valid. We suggest that the disorganised thymic cortical environment in thymoma as well as the absence of thymus after thymectomy might merely fail to delete or tolerate potentially self-reactive T-cells. This may result in a condition where, in the patient with the non-totally disorganised thymus, the symptoms of immunodeficiency do not manifest themselves and appear after thymectomy. This also indicates a possible role of thymus in autoreactive cell elimination in adults $[5,6,9]$.

Furthermore, an accurate and timely diagnosis of immunodeficiency associated with thymoma is important in preventing complications, which may result in irreversible lung tissue destruction. Recurrent infections of the upper and lower respiratory tract as well as sinusitis caused by Haemophilus influenzae, Pneumocystis carinii, Candida albicans, and cytomegalovirus (in our patient - Citrobacter braakii) were reported in most cases. The development of bronchiectasis was also quite frequent in GS. The infection treatment with antibiotics as well as IVIG replacement can be administered as an outpatient therapy every 2-6 weeks depending on immunoglobulin levels [2, $3,17,18]$.

Patients with thymoma should be regularly monitored of the respiratory system function by means of for example chest X-ray and microbiological analysis of opportunistic infections. Patients after thymectomy and with diagnosed immunodeficiency are at an increased risk of complications which may cause irreversible lung damage. Therefore, regular annual cytometric analysis of the lymphocyte subpopulations and biochemical immunoglobulin level as well as careful monitoring of the immunological system status of such patients are required [9].

\section{References}

1. Good RA (1954): Agammaglobulinemia: a provocative experiment of nature. Bull Univ Minn Hosp Med Found 26: 1-19.

2. Joven MH, Palalay MP, Sonido CY (2013): Case report and literature review on Good's syndrome, a form of acquired immunodeficiency associated with thymomas. Hawaii J Med Public Health 72: 56-62.

3. Kelleher P, Misbah SA (2003): What is Good's syndrome? Immunological abnormalities in patients with thymoma. J Clin Pathol 56: 12-16.

4. Fijolek J, Wiatr E, Demkow U, Orlowsk TM (2009): Immunological disturbances in Good's syndrome. Clin Invest Med 32: E301-306

5. Ma D, Wei Y, Liu F (2011): Regulatory mechanisms of thymus and T cell development. Dev Comp Immunol 39: 91-102.

6. Sakaguchi S, Sakaguchi N, Shimizu J (2001): Immunologic tolerance maintained by $\mathrm{CD} 25+\mathrm{CD} 4+$ regulatory T cells: their common role in controlling autoimmunity, tumor immunity, and transplantation tolerance. Immunol Rev 182: 18-32.

7. Weissferdt A, Moran CA (2013): Staging of primary mediastinal tumors. Adv Anat Pathol 20: 1-9.

8. Rashid OM, Cassano AD, Takabe K (2013): Thymic neoplasm: a rare disease with a complex clinical presentation. J Thorac Dis 5: 173-183.

9. Krawczyk P, Adamczyk-Korbel M, Kieszko R, et al. (2007): Immunological system status and the appearance of respiratory system disturbances in thymectomized patients. Arch Immunol Ther Exp (Warsz) 55: 49-56.

10. Kuribayashi K, Fujimi A, Kobune M, et al. (2010): Pure red cell aplasia associated with Good's syndrome accompanied by decreased stem cell factor production in the bone marrow. Intern Med 49: 377-382.

11. Chen J, Yang Y, Zhu D, et al. (2011): Thymoma with pure red cell aplasia and Good's syndrome. Ann Thorac Surg 91: 1620-1622.

12. Taniguchi T, Usami N, Kawaguchi K, Yokoi K (2009): Good syndrome accompanied by pure red cell aplasia. Interact Cardiovasc Thorac Surg 9: 750-752.

13. Waldmann TA, Broder S, Durm M, et al. (1975): Suppressor $\mathrm{T}$ cells in the pathogenesis of hypogammaglobulinemia associated with thymoma. Trans Assoc Am Physicians 88: 120-134.

14. Waldmann TA, Broder S, Krakauer R, et al. (1976): The role of suppressor cells in the pathogenesis of common variable hypogammaglobulinemia and the immunodeficiency associated with myeloma. Fed Prod 35: 2067-2072. 
15. Webster AD (1979): Combined immunodeficiency and thymic abnormalities. J Clin Pathol Suppl (R Coll Pathol) 13: 10-14.

16. Kikuchi R, Mino N, Okamoto T, et al. (2011): A case of Good's syndrome: a rare acquired immunodeficiency associated with thymoma. Ann Thorac Cardiovasc Surg 17: 74-76.

17. Agarwal S, Cunningham-Rundles C (2007): Thymoma and immunodeficiency (Good syndrome): a report of 2 unusual cases and review of the literature. Ann Allergy Asthma Immunol 98: 185-190.

18. Méan M, Schaller MD, Asner S, et al. (2009): Thymoma, immunodeficiency, and herpes simplex virus infections. Med Mal Infect 39: 344-347. 